CERN-TH/98-208

hep-ph/9806440

\title{
Atmospheric and Solar Neutrinos with a Heavy Singlet
}

\author{
S. F. King 円 \\ Theory Division, CERN, CH-1211 Geneva 23, Switzerland.
}

\begin{abstract}
We follow a minimalistic approach to neutrino masses, by introducing a single heavy singlet $N$ into the standard model (or supersymmetric standard model) with a heavy Majorana mass $M$, which couples as a single right-handed neutrino in a Dirac fashion to leptons, and induces a single light see-saw mass $m_{\nu} \sim 5 \times 10^{-2} \mathrm{eV}$, leaving two neutrinos massless. This trivial extension to the standard model may account for the atomospheric neutrino data via $\nu_{\mu} \rightarrow \nu_{\tau}$ oscillations with near maximal mixing angle $\theta_{23} \sim \pi / 4$ and $\Delta m_{\mu \tau}^{2} \sim 2.5 \times 10^{-3} e V^{2}$. In order to account for the solar neutrino data the model is extended to SUSY GUT/ string-inspired type models which can naturally yield an additional light tau neutrino mass $m_{\nu_{\tau}} \sim f e w \times 10^{-3} \mathrm{eV}$ leading to $\nu_{e L} \rightarrow\left(\cos \theta_{23} \nu_{\mu L}-\sin \theta_{23} \nu_{\tau L}\right)$ oscillations with $\Delta m_{e 1}^{2} \sim 10^{-5} e V^{2}$ and a mixing angle $\sin ^{2} 2 \theta_{1} \approx 10^{-2}$ in the correct range for the small angle MSW solution to the solar neutrino problem. The model predicts $\nu_{e L} \rightarrow\left(\sin \theta_{23} \nu_{\mu L}+\cos \theta_{23} \nu_{\tau L}\right)$ oscillations with a similar angle but a larger splitting $\Delta m_{e 2}^{2} \sim 2.5 \times 10^{-3} \mathrm{eV}^{2}$.
\end{abstract}

CERN-TH/98-208

October 13, 2018

\footnotetext{
${ }^{1}$ On leave of absence from Department of Physics and Astronomy, University of Southampton, Southampton, SO17 1BJ, U.K.
} 
Atmospheric neutrino data from Super-Kamiokande [4] and SOUDAN [2], when combined with the recent CHOOZ data [3], are consistent with $\nu_{\mu} \rightarrow \nu_{\tau}$ oscillations with near maximal mixing and $\Delta m_{\mu \tau}^{2} \sim 2.5 \times 10^{-3} \mathrm{eV}^{2}$. The data are equally consistent if $\nu_{\tau}$ is replaced by a light sterile neutrino, and indeed many authors have considered adding an extra light singlet neutrino state, in addition to the usual three heavy singlet neutrinos which accompany the three fermion families in quark-lepton unified models [4]. Without a light sterile neutrino, in the framework of some unified theory, one is faced with the problem of specifying the heavy Majorana matrix which is highly model-dependent and requires additional assumptions. In many cases even with such additional assumptions it is very difficult to account for atmospheric oscillations within the framework of a complete description of quark and lepton masses [5]. (2)

In this paper we shall take a different tack. In order to overcome the problem of the unknown and model-dependent heavy Majorana matrix, we shall begin by assuming it does not exist. More precisely we shall assume that the three "right-handed neutrinos" (or the equivalent string-related physics possibly involving further singlet states which can lead to low energy operators capable of giving light effective Majorana masses) have Majorana masses equal to the GUT scale $M_{R} \sim M_{G U T}$ leading to a third family neutrino mass $m_{\nu_{\tau}} \sim$ few $\times 10^{-3} \mathrm{eV}$, with presumably smaller masses for the first and second families. Since the atmospheric neutrino is heavier than these masses we shall ignore such masses to begin with. In order to account for the atmospheric neutrino data we shall take a minimalistic approach and introduce below the GUT scale only a single heavy singlet $N$ into the standard model (or supersymmetric standard model) with a heavy Majorana mass $M \bar{N} N^{c}$ where $M<M_{G U T}$, which couples as a single right-handed neutrino in a Dirac fashion to leptons, and induces a single light see-saw

\footnotetext{
${ }^{2}$ It is however rather straightforward to envisage scenarios in which the large 23 mixing occurs via the heavy Majorana matrix [6]. It remains to be seen whether such approaches can be incorporated into complete models however.
} 
mass $m_{\nu} \sim 5 \times 10^{-2} \mathrm{eV}$, leaving two neutrinos massless.

To be exact the new singlet $N$ couples to the three lepton doublets $L_{e, \mu, \tau}$ and the Higgs doublet $H_{2}$ (the subscript follows the usual notation of the minimal supersymmetric standard model, or MSSM, but the scheme also applies to the standard model with a single Higgs doublet) as:

$$
\left(\lambda_{e} L_{e}+\lambda_{\mu} L_{\mu}+\lambda_{\tau} L_{\tau}\right) N^{c} H_{2}
$$

where the subscripts $e, \mu, \tau$ indicate that we are in the charged lepton mass eigenstate basis, e.g. $L_{e}=\left(\nu_{e L}, e_{L}^{-}\right)$where $e^{-}$is the electron mass eigenstate and $\nu_{e}$ is the associated neutrino weak eigenstate, and $\lambda_{e}$ is a Yukawa coupling to the $\operatorname{singlet} N$ in this basis. When the Higgs develops its low energy vacuum expectation value $v_{2}$ Eq.1] will lead to Dirac neutrino masses

$$
v_{2} \bar{N}_{R}\left(\lambda_{e} \nu_{e L}+\lambda_{\mu} \nu_{\mu L}+\lambda_{\tau} \nu_{\tau L}\right)
$$

corresponding to a single Dirac mass eigenstate

$$
\nu_{L}=\frac{1}{\sqrt{\left|\lambda_{e}\right|^{2}+\left|\lambda_{\mu}\right|^{2}+\left|\lambda_{\tau}\right|^{2}}}\left(\lambda_{e} \nu_{e L}+\lambda_{\mu} \nu_{\mu L}+\lambda_{\tau} \nu_{\tau L}\right)
$$

with a Dirac mass

$$
m_{\nu}^{\text {Dirac }}=\sqrt{\left|\lambda_{e}\right|^{2}+\left|\lambda_{\mu}\right|^{2}+\left|\lambda_{\tau}\right|^{2}} v_{2}
$$

Of course the singlet $N$ has a very large Majorana mass $M$, so the physical mass is the light effective neutrino Majorana mass given by the see-saw mechanism as:

$$
m_{\nu}=\frac{m_{\nu}^{\text {Dirac }^{2}}}{M}=\left(\left|\lambda_{e}\right|^{2}+\left|\lambda_{\mu}\right|^{2}+\left|\lambda_{\tau}\right|^{2}\right) \frac{v_{2}^{2}}{M}
$$

Clearly the light effective Majorana neutrino eigenstate $\nu_{L}$ is the same as the Dirac neutrino eigenstate in Eq.3.

In order to explain the atmospheric neutrino data we shall assume that the three Yukawa couplings, which are completely free parameters in this model, satisfy the 
relation:

$$
\lambda_{e} \ll \lambda_{\mu} \approx \lambda_{\tau}
$$

In this case the mass eigenstate neutrino can be expanded in terms of the three weak eigenstate neutrinos in an approximate form involving a small angle $\theta_{1}$ and a large angle $\theta_{23}$ as:

$$
\nu_{L}=\theta_{1} \nu_{e L}+s_{23} \nu_{\mu L}+c_{23} \nu_{\tau L}
$$

which by comparison with Eq.3 implies

$\theta_{1} \approx \frac{\lambda_{e}}{\sqrt{\left|\lambda_{e}\right|^{2}+\left|\lambda_{\mu}\right|^{2}+\left|\lambda_{\tau}\right|^{2}}}, s_{23} \approx \frac{\lambda_{\mu}}{\sqrt{\left|\lambda_{e}\right|^{2}+\left|\lambda_{\mu}\right|^{2}+\left|\lambda_{\tau}\right|^{2}}}, c_{23} \approx \frac{\lambda_{\tau}}{\sqrt{\left|\lambda_{e}\right|^{2}+\left|\lambda_{\mu}\right|^{2}+\left|\lambda_{\tau}\right|^{2}}}$

where $s_{23}=\sin \theta_{23}, c_{23}=\cos \theta_{23}$.

The interpretation of the atmospheric neutrino mixing is now clear. There is a single massive neutrino $\nu_{L}$ with a Majorana mass $m_{\nu} \sim 5 \times 10^{-2} \mathrm{eV}$, which contains large and approximately equal components of the weak eigenstates $\nu_{\mu L}$ and $\nu_{\tau L}$ parametrised by the mixing angle $\theta_{23} \sim \pi / 4$. There will also be a small admixture of the weak eigenstate $\nu_{e L}$ in the mass eigenstate $\nu_{L}$, parametrised by the angle $\theta_{1}$ according to Eq.7. The two neutrino eigenstates which are orthogonal to $\nu_{L}$ will be massless. If we set $\theta_{1}=0$ then one of the massless neutrinos will be $\nu_{e L}$ and the other will be the orthogonal combination

$$
\nu_{0 L} \approx c_{23} \nu_{\mu L}-s_{23} \nu_{\tau L}
$$

In this approximation the atmospheric neutrino data is then consistent with $\nu_{\mu} \rightarrow \nu_{\tau}$ oscillations via two state mixing involving the massless neutrino $\nu_{0 L}$ in Eq.9 and the massive neutrino $\nu_{L}$ in Eq.7 with mass $m_{\nu} \sim 5 \times 10^{-2} \mathrm{eV}$.

So far we have provided a simple explanation of the atomospheric neutrino data, assuming that $\theta_{1} \ll \theta_{23} \sim \pi / 4$. We shall show that by including a single extra Majorana mass for the tau neutrino $m_{\nu_{\tau}} \sim f e w \times 10^{-3} \mathrm{eV}$ we can also account for the 
solar neutrino data via the small angle MSW effect [7]. Since $m_{\nu_{\tau}} \ll m_{\nu}$ the presence of such a mass in the $\nu_{\tau L}$ component of $\nu_{L}$ will only slightly perturb the mass of this state at the $10 \%$ level. However turning to the two orthogonal neutrinos, still assuming that $\theta_{1}=0$, the state $\nu_{0 L}$ in Eq.9 will clearly pick up a mass of order $s_{23}^{2} m_{\nu_{\tau}} \sim m_{\nu_{\tau}} / 2 \sim 3 \times 10^{-3} \mathrm{eV}$ (say) via its $\nu_{\tau L}$ component, while $\nu_{e L}$ will remain massless. When $\theta_{1}$ is switched on the weak eigenstate $\nu_{e L}$ will consist dominantly of the massless state, plus a small admixture of both the lighter and heavier mass eigenstates, with the lighter of the two relevant for the MSW solution of the solar neutrino problem.

We shall now discuss in more detail the structure of the mixing matrix in the presence of a finite mixing angle $\theta_{1}$ and a non-zero tau neutrino mass $m_{\nu_{\tau}}$. Taking $\theta_{1} \neq 0$ and small, but keeping $m_{\nu_{\tau}}=0$ to start with, the mass spectrum of neutrinos consists of one massive state $\nu_{L}$ in Eq.7 with mass $m_{\nu}$ in Eq.5, with two orthogonal combinations $\nu_{e L}^{\prime}$ and $\nu_{0 L}^{\prime}$ corresponding to two massless neutrinos. The charged weak currents in the standard model are:

$$
W_{\mu}^{-}(\bar{e}, \bar{\mu}, \bar{\tau})_{L} \gamma^{\mu}\left(\begin{array}{c}
\nu_{e} \\
\nu_{\mu} \\
\nu_{\tau}
\end{array}\right)_{L}+\text { h.c. }
$$

where $\nu_{e L}, \nu_{\mu L}, \nu_{\tau L}$ are neutrino weak eigenstates which couple with unit strength to $e, \mu, \tau$, respectively. The mixing matrix $U$ is defined by the unitary transformation which relates the weak eigenstates to the mass eigenstates:

$$
\left(\begin{array}{l}
\nu_{e} \\
\nu_{\mu} \\
\nu_{\tau}
\end{array}\right)_{L}=U\left(\begin{array}{l}
\nu_{e}^{\prime} \\
\nu_{0}^{\prime} \\
\nu
\end{array}\right)_{L}
$$

Since there is a degeneracy in the massless neutrino subspace, the mixing matrix $U$ is only determined up to an overall rotation in the two-dimensional massless subspace. Since the degeneracy will ultimately be lifted by $m_{\nu_{\tau}}$ (assuming that the first and second family GUT generated masses are negligible) it is convenient to rotate to a massless basis where $\nu_{e L}^{\prime}$ does not contain any component of $\nu_{\tau L}$, and so will remain 
massless when $m_{\nu_{\tau}}$ is eventually switched on. In such a basis the mixing matrix is uniquely specified, and to first order in $\theta_{1}$ is:

$$
U=\left(\begin{array}{ccc}
1 & \left(\frac{c_{23}}{s_{23}}\right) \theta_{1} & \theta_{1} \\
-\frac{\theta_{1}}{s_{23}} & c_{23} & s_{23} \\
0 & -s_{23} & c_{23}
\end{array}\right)
$$

In this basis the mass eigenstates expressed in terms of the weak eigenstates are summarised below:

$$
\begin{aligned}
\nu_{e L}^{\prime} & =\nu_{e L}-\frac{\theta_{1}}{s_{23}} \nu_{\mu L} \\
\nu_{0 L}^{\prime} & =\left(\frac{c_{23}}{s_{23}}\right) \theta_{1} \nu_{e L}+c_{23} \nu_{\mu L}-s_{23} \nu_{\tau L} \\
\nu_{L} & =\theta_{1} \nu_{e L}+s_{23} \nu_{\mu L}+c_{23} \nu_{\tau L}
\end{aligned}
$$

In the $\theta_{1}=0$ limit $\nu_{e L}^{\prime}, \nu_{0 L}^{\prime}$ return to $\nu_{e L}, \nu_{0 L}$ defined previously.

Now we consider the effect of a non-zero $m_{\nu_{\tau}}$. Because of our judicious choice of basis the state $\nu_{e L}^{\prime}$ will remain massless because it does not have any tau neutrino component. The only effect will be to re-mix the states $\nu_{0 L}^{\prime}$ and $\nu_{L}$, inducing a mass matrix in this sector of:

$$
\left(\begin{array}{ll}
s_{23}^{2} m_{\nu_{\tau}} & -s_{23} c_{23} m_{\nu_{\tau}} \\
-s_{23} c_{23} m_{\nu_{\tau}} & c_{23}^{2} m_{\nu_{\tau}}+m_{\nu}
\end{array}\right)
$$

Now $m_{\nu_{\tau}} / 2 \sim 3 \times 10^{-3} \mathrm{eV}$ is an order of magnitude smaller than $m_{\nu} \sim 5 \times 10^{-2} \mathrm{eV}$ so we expect additional mixing between $\nu_{0 L}^{\prime}$ and $\nu_{L}$ parametrised by a small angle $\theta_{\tau} \sim s_{23} c_{23} \frac{m_{\nu_{\tau}}}{m_{\nu}}$. With $m_{\nu_{\tau}}$ switched on we continue to denote the mass eigenstates as $\nu_{0 L}^{\prime}, \nu_{L}$ as in Eq.11 with the modified mass eigenvalues:

$$
\begin{aligned}
m_{\nu_{0 L}^{\prime}} & \approx s_{23}^{2} m_{\nu_{\tau}} \sim 3 \times 10^{-3} \mathrm{eV} \\
m_{\nu_{L}} & \approx m_{\nu}+c_{23}^{2} m_{\nu_{\tau}} \sim 5 \times 10^{-2} \mathrm{eV}
\end{aligned}
$$

leaving $\nu_{e L}^{\prime}$ massless. We continue to denote the mixing matrix as $U$ which is now 
modified from its form in Eq.12 as follows:

$$
U=\left(\begin{array}{ccc}
1 & \left(\frac{c_{23}}{s_{23}}\right) \theta_{1} & \theta_{1} \\
-\frac{\theta_{1}}{s_{23}} & c_{23}\left(1+s_{23}^{2} \frac{m_{\nu_{\tau}}}{m_{\nu}}\right) & s_{23}\left(1-c_{23}^{2} \frac{m_{\nu_{\tau}}}{m_{\nu}}\right) \\
0 & -s_{23}\left(1-c_{23}^{2} \frac{m_{\nu_{\tau}}}{m_{\nu}}\right) & c_{23}\left(1+s_{23}^{2} \frac{m_{\nu \tau}}{m_{\nu}}\right)
\end{array}\right)
$$

The exact masses and mixing angles are given in the Appendix.

The physics of atmospheric neutrinos may be summarised as follows. The weak eigenstate $\nu_{\mu L}$ may be expanded in terms of the mass eigenstates as:

$$
\nu_{\mu L}=-\frac{\theta_{1}}{s_{23}} \nu_{e L}^{\prime}+c_{23}\left(1+s_{23}^{2} \frac{m_{\nu_{\tau}}}{m_{\nu}}\right) \nu_{0 L}^{\prime}+s_{23}\left(1-c_{23}^{2} \frac{m_{\nu_{\tau}}}{m_{\nu}}\right) \nu_{L}
$$

There will be atmospheric neutrino oscillations $\nu_{\mu} \rightarrow \nu_{\tau}$ where maximal mixing now corresponds to $\tan \theta_{23} \approx 1+\frac{m_{\nu_{\tau}}}{m_{\nu}}$, with

$$
\Delta m_{\mu \tau}^{2}=\left(m_{\nu}+m_{\nu_{\tau}}\left(c_{23}^{2}-s_{23}^{2}\right)\right)^{2} \approx m_{\nu}^{2} \sim 2.5 \times 10^{-3} e V^{2}
$$

The weak eigenstate $\nu_{\mu L}$ will also mix slightly with $\nu_{e L}$ but since $\theta_{1} \ll \theta_{23}$ this mixing will not be noticed by the atmospheric neutrino experiments.

The situation regarding $\nu_{e L}$ mixing is quite interesting. The weak eigenstate $\nu_{e L}$ may be expanded in terms of the mass eigenstates as:

$$
\nu_{e L}=\nu_{e L}^{\prime}+\left(\frac{c_{23}}{s_{23}}\right) \theta_{1} \nu_{0 L}^{\prime}+\theta_{1} \nu_{L}
$$

An electron neutrino weak eigenstate $\nu_{e L}$ will therefore have a dominant massless component $\nu_{e L}^{\prime}$ which is split from the massive states by two different mass splittings:

$$
\begin{aligned}
& \Delta m_{e 1}^{2}=\left(m_{\nu_{0 L}^{\prime}}-m_{\nu_{e L}^{\prime}}\right)^{2}=m_{\nu_{0 L}^{\prime}}^{2} \sim 10^{-5} e V^{2} \\
& \Delta m_{e 2}^{2}=\left(m_{\nu_{L}}-m_{\nu_{e L}^{\prime}}\right)^{2}=m_{\nu_{L}}^{2} \sim 2.5 \times 10^{-3} e V^{2}
\end{aligned}
$$

with the physics of electron neutrino oscillations governed by Eqs.19 and 20. Electron neutrino oscillations can be roughly characterised as follows:

$$
\nu_{e L} \rightarrow \nu_{0 L}^{\prime} \approx c_{23} \nu_{\mu L}-s_{23} \nu_{\tau L}
$$


with $\Delta m_{e 1}^{2}$ and a mixing amplitude $\left(\frac{c_{23}}{s_{23}}\right) \theta_{1}$. In addition we expect oscillations to the orthogonal combination:

$$
\nu_{e L} \rightarrow \nu_{L} \approx s_{23} \nu_{\mu L}+c_{23} \nu_{\tau L}
$$

with $\Delta m_{e 2}^{2}$ and a mixing amplitude $\theta_{1}$. Assuming that $s_{23} \approx c_{23}$, then the solar neutrino problem may be solved by the small angle MSW solution with $\sin ^{2} 2 \theta_{1} \sim 10^{-2}$, in which case the oscillations with $\Delta m_{e 1}^{2} \sim 10^{-5} e V^{2}$ will be resonantly enhanced. The orthogonal oscillations with $\sin ^{2} 2 \theta_{1} \sim 10^{-2}$ and $\Delta m_{e 2}^{2} \sim 2.5 \times 10^{-3} e V^{2}$ are not resonantly enhanced and play only a negligible role in solar physics, however eventually one might hope to observe such oscillations in vacuo. The new generation of solar neutrino experiments will be able to measure the mixing angle $\theta_{23}$ by counting the number of $\nu_{\mu L}$ and $\nu_{\tau L}$ events which will be equal in the case of maximal atmospheric mixing.

We now give a brief comparison to recent approach which has some superficial similarities to our scheme [8]. The addition of a single gauge singlet $N$ with Yukawa couplings in Eq.a gives rise to a light effective Majorana matrix in the $\nu_{e L}, \nu_{\mu L}, \nu_{\tau L}$ basis proportional to

$$
\left(\begin{array}{lll}
\lambda_{e}^{2} & \lambda_{e} \lambda_{\mu} & \lambda_{e} \lambda_{\tau} \\
\lambda_{e} \lambda_{\mu} & \lambda_{\mu}^{2} & \lambda_{\mu} \lambda_{\tau} \\
\lambda_{e} \lambda_{\tau} & \lambda_{\mu} \lambda_{\tau} & \lambda_{\tau}^{2}
\end{array}\right) \frac{v_{2}^{2}}{M}
$$

assuming for simplicity that the Yukawa couplings are real. Such a matrix is similar to that proposed recently [8], and clearly gives rise to one massive neutrino and two massless neutrinos. We have simply assumed that $\lambda_{e} \ll \lambda_{\mu} \sim \lambda_{\tau}$ in order to account for the atmospheric neutrino results, but clearly such a structure could be enforced by suitable (approximate) discrete symmetries. However whereas in our model the basic structure in Eq.23 is a simple consequence of having a single gauge singlet, in the other model it is due to R-parity violating couplings with certain discrete symmetries [8]. We have shown that a small perturbation in the 33 element of the matrix in Eq.23 coresponding to a tau neutrino light Majorana mass of $m_{\nu_{\tau}}$ coming from GUT or 
string scale physics can give a small mass to one of the previously massless eigenstates, leaving the other one massless, and have used this small splitting to account for the solar neutrino data. There will of course be other contributions to the mass matrix coming from GUT or string scale masses involving the first and second families, but they are expected to be much smaller than $m_{\nu_{\tau}}$ according to general arguments, and we have ignored them here. By contrast the perturbations to the other elements of

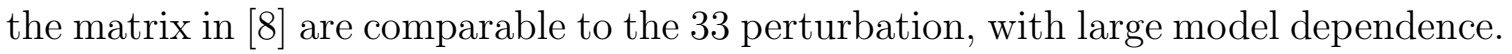
This implies that our simple predictions for electron neutrino oscillations discussed above will not be shared by the other model $[8]$.

As regards the origin of the singlet $N$ we remark that our model may be embedded into an $S O(10)$ type model if the singlet $N$ originates from some new $16^{\prime}$ which is part of a vector representation $16^{\prime}+\overline{16}^{\prime}$ with a heavy vector mass $M_{V} 16^{\prime} \overline{1}^{\prime}$ which may be comparable to the Majorana mass $M 16^{\prime} 16^{\prime} \rightarrow M N N$. The standard couplings $16_{i} 16_{j} 10$ are then supplemented by new couplings $16_{i} 16^{\prime} 10$ which include the Dirac neutrino couplings to the singlet $N$ contained in the $16^{\prime}$. It will also include additional Dirac couplings which mix charged quarks and leptons with the new exotic quarks and leptons contained in the $16^{\prime}$. However the new exotic quarks and leptons all have large vector masses $M_{V}$, so such couplings give a negligible contribution to charged quark and lepton masses and mixing angles. The standard "right-handed neutrinos" contained in the $16_{i}$ 's are all assumed to have GUT scale masses in such a scenario, leading to the small tau neutrino mass perturbation that we need for the solar neutrino problem. There may of course be other origins for the singlet $N$ which have nothing to do with $S O(10)$.

Finally we remark that with our neutrino spectrum consisting of two neutrinos of mass $5 \times 10^{-2} \mathrm{eV}$ and $3 \times 10^{-3} \mathrm{eV}$ and one massless (or strictly very light) neutrino, it is not possible to use this model to account for hot dark matter. We also cannot account for the LSND data [9]. If either becomes mandatory then the scheme described here 
would be excluded.

\section{Acknowledgements}

I would like to thank Steve Abel, Sacha Davidson and Graham Ross for useful comments.

\section{Appendix: Exact Masses and Mixing Angles}

There are 5 underlying parameters as defined in the text:

$$
\lambda_{e}, \lambda_{\mu}, \lambda_{\tau}, M, m_{\nu_{\tau}}
$$

which are assumed to all take real values. From these 5 parameters we obtain 4 physical observables, consisting of 2 masses $m_{\nu_{\tau}}$ and $m_{\nu}$ defined in Eq.5, plus 2 angles defined as:

$$
\begin{aligned}
s_{1} \equiv \sin \theta_{1} & \equiv \frac{\lambda_{e}}{\sqrt{\left|\lambda_{e}\right|^{2}+\left|\lambda_{\mu}\right|^{2}+\left|\lambda_{\tau}\right|^{2}}} \\
t_{23} & \equiv \tan \theta_{23} \equiv \frac{\lambda_{\mu}}{\lambda_{\tau}}
\end{aligned}
$$

The exact expressions for neutrino masses and mixing angles are then expressed in terms of $\theta_{1}, \theta_{23}, m_{\nu}, m_{\nu_{\tau}}$.

The mixing matrix $U$ is defined by the unitary transformation which relates the weak eigenstates to the mass eigenstates:

$$
\left(\begin{array}{l}
\nu_{e} \\
\nu_{\mu} \\
\nu_{\tau}
\end{array}\right)_{L}=U\left(\begin{array}{c}
\nu_{e}^{\prime} \\
\nu_{0}^{\prime} \\
\nu
\end{array}\right)_{L}
$$

The exact mass eigenvalues are:

$$
\begin{aligned}
& m_{\nu_{e L}^{\prime}}=0 \\
& m_{\nu_{0 L}^{\prime}}=\delta_{11} c_{\tau}^{2}-2 \delta_{12} c_{\tau} s_{\tau}+\left(\delta_{22}+m_{\nu}\right) s_{\tau}^{2} \\
& m_{\nu_{L}}=\left(\delta_{22}+m_{\nu}\right) c_{\tau}^{2}+2 \delta_{12} c_{\tau} s_{\tau}+\delta_{11} s_{\tau}^{2}
\end{aligned}
$$


where

$$
\begin{aligned}
& \delta_{11}=s_{23}^{2}\left(1+\frac{s_{1}^{2}}{t_{23}^{2}}\right)^{1 / 2} m_{\nu_{\tau}} \\
& \delta_{22}=c_{23}^{2} c_{1}^{2} m_{\nu_{\tau}} \\
& \delta_{12}=s_{23} c_{23} c_{1}\left(1+\frac{s_{1}^{2}}{t_{23}^{2}}\right)^{1 / 2} m_{\nu_{\tau}}
\end{aligned}
$$

and $s_{\tau}=\sin \theta_{\tau}, c_{\tau}=\cos \theta_{\tau}$ where $\theta_{\tau}$ is the additional mixing angle induced by $m_{\nu_{\tau}}$ and is given by

$$
\tan 2 \theta_{\tau}=\frac{2 \delta_{12}}{m_{\nu}+\delta_{22}-\delta_{11}}
$$

The exact mixing matrix is:

$$
U=\left(\begin{array}{ccc}
\left(1+\frac{t_{1}^{2}}{s_{23}^{2}}\right)^{-\frac{1}{2}} & \frac{c_{\tau} s_{1}}{t_{23}}\left(1+\frac{t_{1}^{2}}{s_{23}^{2}}\right)^{-\frac{1}{2}}+s_{\tau} s_{1} & c_{\tau} s_{1}-\frac{s_{\tau} s_{1}}{t_{23}}\left(1+\frac{t_{1}^{2}}{s_{23}^{2}}\right)^{-\frac{1}{2}} \\
-\frac{t_{1}}{s_{23}}\left(1+\frac{t_{1}^{2}}{s_{23}^{2}}\right)^{-\frac{1}{2}} & c_{\tau} c_{23} c_{1}^{2}\left(1+\frac{s_{1}^{2}}{t_{23}^{2}}\right)^{-\frac{1}{2}}+s_{\tau} s_{23} c_{1} & c_{\tau} s_{23} c_{1}-s_{\tau} c_{23} c_{1}^{2}\left(1+\frac{s_{1}^{2}}{t_{23}^{2}}\right)^{-\frac{1}{2}} \\
0 & -c_{\tau} s_{23}\left(1+\frac{s_{1}^{2}}{t_{23}^{2}}\right)^{\frac{1}{2}}+s_{\tau} c_{23} c_{1} & c_{\tau} c_{23} c_{1}+s_{\tau} s_{23}\left(1+\frac{s_{1}^{2}}{t_{23}^{2}}\right)^{\frac{1}{2}}
\end{array}\right)
$$

\section{References}

[1] Y. Fukuda et al, hep-ex/9805006, hep-ex/9805021, hep-ex/9803006.

[2] S. M. Kashara et al, Phys. Rev. D 55 (1997) 5282.

[3] M. Apollonio et al, Phys. Lett. B420 (1998) 397.

[4] V. Barger et al, Phys. Rev. Lett. 45 (1980) 962;

D. Caldwell and R. Mohapatra, Phys. Rev. D 48 (1993) 3259;

J. Peltoniemi and J. Valle, Nucl. Phys. B406 (1993) 409;

R. Foot and R. Volkas, Phys. Rev. D52 (1995) 6595;

E. Ma and P. Roy, Phys. Rev. D52 (1995) R4780;

Z. Berezhiani and R. Mohapatra, Phys. Rev. D 52 (1995) 6607;

E. Chun et al, Phys. Lett. B357 (1995) 608. J. Espinosa, hep-ph/9707541; 
P. Langacker, hep-ph/9805281;

A. S. Joshipura and A. Yu. Smirnov, hep-ph/9806376.

[5] H. Dreiner et al, Nucl. Phys. B436 (1995) 461.

[6] B. Allanach, hep-ph/9806294;

G. Leontaris et al, Phys. Rev. D 53 (1996) 6381.

[7] L. Wolfenstein, Phys. Rev. D17 (1978) 2369;

S. Mikheyev and A. Yu. Smirnov, Sov. J. Nucl. Phys. 42 (1985) 913.

[8] M. Drees et al, Phys. Rev. D57 (1998) 5335.

[9] C. Athanassapoulos et al, Phys. Rev. C54 (1996) 2685. 